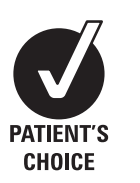

CHOICE

\title{
Epidemiology and pathophysiology of falls in facioscapulohumeral disease
}

\author{
C G C Horlings, ${ }^{1,2}$ M Munneke, ${ }^{1}$ A Bickerstaffe, ${ }^{1}$ L Laverman, ${ }^{1} \mathrm{~J}$ H J Allum, ${ }^{2}$ \\ G W A M Padberg, ${ }^{1}$ B R Bloem, ${ }^{1}$ B G M van Engelen'
}

\begin{abstract}
${ }^{1}$ Radboud University Nijmegen Medical Centre, Donders Institute for Brain, Cognition and Behaviour, Department of Neurology, The Netherlands;

${ }^{2}$ Department of ORL, University Hospital Basel, Switzerland
\end{abstract}

Correspondence to:

Professor B R Bloem,

Department of Neurology (HP

935), Donders Centre for Brain,

Cognition and Behaviour,

Radboud University Nijmegen

Medical Centre, PO Box 9101

6500 HB Nijmegen, The

Netherlands;

B.Bloem@neuro.umcn.nl

Received 2 February 2009

Revised 29 April 2009

Accepted 30 April 2009

Published Online First

21 June 2009

\section{ABSTRACT}

Background and aim: Muscle weakness is a potentially important, yet poorly studied, risk factor for falls. Detailed studies of patients with specific myopathies may shed new light on the relation between muscle weakness and falls. Here falls in patients with facioscapulohumeral disease (FSHD) who suffered from lower limb muscle weakness were examined. This study provides insights into the prevalence, relevance and pathophysiology of falls in FSHD.

Methods: A validated questionnaire was used as well as a prospective 3 month follow-up to examine the prevalence, circumstances and consequences of falls in 73 patients with FSHD and 49 matched healthy controls. In a subgroup of 28 subjects, muscle strength was also examined and balance was assessed electrophysiologically using body worn gyroscopes.

Results: In the questionnaire, 30\% of the patients reported falling at least once a month whereas none of the controls did. Injuries occurred in almost $70 \%$ of the patients. The prospective study showed that patients fell mostly at home, mainly due to intrinsic (patient related) causes, and usually in a forward direction. Fallers were unstable while climbing stairs, rising from a chair and standing with eyes closed whereas non-fallers had normal balance control. Frequent fallers had greater muscle weakness than infrequent fallers.

Conclusion: These findings demonstrate the high prevalence and clinical relevance of falls in FSHD. The relation between muscle weakness and instability among fallers is also highlighted. Because patients fell mainly at home, fall prevention strategies should focus on home adaptations. As mainly intrinsic causes underlie falls, the impact of adopting balance strategies or balance training should be explored in this patient group.

In patients suffering from neurological diseases, falling is a prevalent and clinically relevant problem. For example, in patients with Parkinson's disease, about 50\% fell at least once during a 6 month follow-up period and 25\% experienced multiple falls. ${ }^{12}$ Comparable fall rates have been reported for patients with other central neurological disorders, such as cerebellar ataxia ${ }^{3}$ or Huntington's disease. ${ }^{4}$ Injuries, fear of falling and an associated reduction of activities are also common among patients with neurological diseases. $^{5}$

Much less is known about fall rates in patients with neuromuscular disorders. In one study, $27 \%$ of patients with a variety of neuromuscular disorders fell during a 3 month follow-up period. Among these, $79 \%$ of fallers sustained minor and $5 \%$ major injuries. ${ }^{6}$ Many patients reported fear of recurrent falling (58\%), which also increases the risk of falls. ${ }^{7}$ A prospective study among patients with myotonic dystrophy showed a 10-fold increase in falls compared with healthy controls. ${ }^{8}$ Among patients with motor neuron disease, 30\% experienced falls. ${ }^{5}$ In a small study among 11 patients with inclusion body myositis, six patients gave a history of falls.?

Developing optimal prevention strategies calls for good understanding of the complex pathophysiology of falls. Patient related risk factors such as cognitive impairment, previous falls, problems with dual tasks and use of sedative medication are examples of generally accepted risk factors for falling. ${ }^{15610}$ More recent work has emphasised muscle weakness as another potentially important, but hitherto poorly studied, risk factor for falls. ${ }^{11}$ Weakness is often reported as a contributing element when present in elderly patients who feature multiple risk factors. But the risk of muscle weakness per se has rarely been studied. Lower extremity weakness increases the risk for recurrent falls threefold in the elderly. ${ }^{12}$ Muscle weakness has also been directly and indirectly associated with falls in post-polio patients. ${ }^{13}$ However, the prevalence and clinical relevance of falls in patients with various neuromuscular disorders featuring muscle weakness remains unclear. ${ }^{11}$ Intentionally, such epidemiological studies in neuromuscular patients could also serve as an "experiment of nature" to provide insights into the pathophysiology of falls.

As a first step, we studied the epidemiology and pathophysiology of falls in patients with facioscapulohumeral dystrophy (FSHD). We chose FSHD for four reasons.

(1) FSHD is the third most common inherited muscular dystrophy..$^{14}$

(2) Our clinical impression is that falls among these patients are common and debilitating but this has never been studied formally.

(3) FSHD is characterised by a specific pattern of weakness. It initially affects the facial, scapular and humeral muscles, then the peroneal muscles in $80 \%$ and pelvic girdle muscles in $20 \%$ of patients. ${ }^{14}$ Thus depending on disease stage and its progression pattern, patients may suffer from severe or minor, proximal, distal or overall weakness, allowing us to study the contribution of the severity and pattern of weakness on fall rates.

(4) Other potential intrinsic risk factors for falling, such as visual deficits or somatosensory disorders, are usually absent in patients with FSHD. ${ }^{14} 1617$ 
Because no cure exists for FSHD, care is essential. Developing fall prevention strategies should be an essential part of this care.

Our approach was bifold. We conducted a combined epidemiological and pathophysiological study that aimed to determine the prevalence, clinical relevance and mechanisms of falling in patients with FSHD. To this end, a validated retrospective questionnaire and a prospective follow-up were conducted. A balance assessment was performed among a subgroup of participants to see whether this could differentiate fallers from non-fallers and provide leads for prevention. A muscle strength assessment was performed to determine the influence of the severity and pattern of muscle weakness on falls. We hypothesised that FSHD patients have a greater fall incidence than healthy controls. The frequency and pathophysiology of falls is expected to be associated with the presence of either or both distal and proximal muscle weakness.

\section{METHODS}

\section{Participants}

A total of 104 adult patients with a definite diagnosis of FSHD were asked to participate. Patients were recruited via the Neuromuscular Centre of the Radboud University Nijmegen Medical Centre and via the Dutch Neuromuscular Diseases Association (Vereniging Spierziekten Nederland, VSN). ${ }^{18} \mathrm{We}$ purposely included patients with a broad spectrum of disease severity because of the exploratory nature of our study. Seventy-seven patients (74\% of the eligible population) agreed to participate in the retrospective part of the study. Six did not return the falls questionnaire and were not included in the retrospective analysis. We also included 47 healthy controls, mainly partners or other carers. Seventy-three patients agreed to participate in the prospective follow-up which was completed by 72 patients (67 patients from the retrospective study plus five more) and 49 controls (47 from the retrospective study plus two more). The only exclusion criteria were the inability to read and write the Dutch language and any psychiatric or cognitive disease. Demographic and anthropometric details of the participants are given in table 1. In a subgroup of the participants, muscle strength and balance were assessed (see also table 1). For these participants, disease severity of FSHD was scored using a 10 grade clinical severity scale ${ }^{19}$ adapted from the Ricci score. ${ }^{20}$ The study was approved by the ethics committee of the Radboud University Nijmegen Medical Centre.

\section{Procedure}

\section{Epidemiology}

A fall was defined as "any unexpected event that caused the person to land unintentionally on any lower surface (floor or object) regardless of any sustained injury". A near fall was defined as "an event where a person lost balance, but prevented falling by regaining balance or seeking external support".

Retrospectively, participants were asked to complete a questionnaire, detailing their current medical condition (including neurological, orthopaedic, balance problems), prior history of falls, near falls and stumbles for the last week, month, year, and fall frequency in general, and the consequences of these falls. Participants also reported the site and (subjective) severity of their muscle weakness. The questionnaire also included questions regarding the functional ability of performing four different tasks: standing on toes and standing on heels requiring mainly distal lower limb strength, and getting up from a chair and walking stairs requiring mainly proximal strength. The questionnaire had been validated in previous studies on falls in patients with neurodegenerative disorders ${ }^{13} 4$ and a pilot study in patients with neuromuscular disorders. ${ }^{6}$ The questionnaire gave us the opportunity to determine fall prevalence, relevance and its relation to muscle weakness over a longer period.

Additionally, participants were contacted by telephone once a week over 13 weeks to register any falls prospectively. The telephone call was delivered by an automatic computer phone system (Pfizer Medconnect, Capelle aan den Ijssel, The Netherlands). All participants received written instructions on how to answer this phone call. The researcher personally contacted the subjects who could not be reached by computer and also approached all subjects who had fallen. During this personal telephone interview, information was gathered about the timing, location, preceding activities, direction, consequences (eg, injuries) and apparent cause of the fall. The follow-up enabled us to collect detailed information on fall circumstances for each fall separately.

\section{Pathophysiology \\ Balance assessment}

Balance was measured in a subgroup of participants (see table 1), including eight frequent fallers (with at least one fall a year according to the questionnaire and at least two falls during the follow-up), nine infrequent fallers (with less than one fall a year according to the questionnaire and a maximum of one fall during the follow-up) and nine healthy controls (with less than one fall a year according to the questionnaire and a maximum of

Table 1 Participants characteristics of prospective fall study

\begin{tabular}{|c|c|c|c|c|c|}
\hline & \multicolumn{2}{|c|}{ Epidemiology } & \multicolumn{3}{|c|}{ Muscle strength and balance assessment } \\
\hline & FSHD & Controls & $\begin{array}{l}\text { FSHD frequent } \\
\text { fallers }\end{array}$ & $\begin{array}{l}\text { FSHD infrequent } \\
\text { fallers }\end{array}$ & Controls \\
\hline $\mathrm{n}$ & 72 & 49 & 8 & 10 & 10 \\
\hline $\operatorname{Sex}(M / F)$ & $42 / 30$ & $20 / 29$ & $5 / 3$ & $6 / 4$ & $5 / 5$ \\
\hline Age (years) & $50(1.2)$ & $50(1.4)$ & $54(2.5)$ & $49(3.0)$ & $56(2.2)$ \\
\hline Height $(\mathrm{cm})$ & $179(1.1)$ & $173(1.4)$ & $173(2.9)$ & $178(3.6)$ & $171(3.1)$ \\
\hline Weight (kg) & $81(1.8)$ & $79(2.4)$ & $80(8.1)$ & $83(5.3)$ & $78(5.5)$ \\
\hline $\mathrm{BMI}\left(\mathrm{kg} / \mathrm{m}^{2}\right)$ & $25(0.5)$ & $26(0.7)$ & $26(2.2)$ & $26(1.4)$ & $27(1.6)$ \\
\hline CSS & - & - & $6.8(0.49)^{* *}$ & $4.9(0.59)^{* *}$ & $0(0)$ \\
\hline CSS range & - & - & $5-9$ & $1-6$ & $0-0$ \\
\hline
\end{tabular}

General characteristics for all participants and separately for those included in the muscle strength assessment. Note that the FSHD frequent fallers, infrequent fallers and controls were similar in age, height, weight and BMI, but not for the CSS.

Values are mean (SEM) unless otherwise indicated.

${ }^{* *} \mathrm{p}<0.01$ compared with controls.

$\mathrm{BMI}$, body mass index; CSS, clinical severity scale; FSHD, facioscapulohumeral disease. 
one fall during follow-up). Balance was measured using body worn gyroscopes ${ }^{21}$ measuring trunk sway in the forwardbackwards (pitch) and sideways (roll) direction during several stance and gait tasks. The system measuring balance control (Swaystar Balance International Innovations $\mathrm{GmbH}$, Switzerland) was connected to a computer via a Bluetooth connection. The stance tasks lasted $20 \mathrm{~s}$ and consisted of standing on firm surface with eyes closed, and standing on a foam support surface with eyes open and eyes closed. The gait tasks included climbing stairs (two steps of height $30 \mathrm{~cm}$ ); walking eight tandem (heel-to-toe) steps; and rising from a chair and walking $3 \mathrm{~m}$. In addition, a timed up and go test (ie, rise from a chair, walk $3 \mathrm{~m}$, turn around and return to a seated position in the chair) was completed without wearing the device. The balance assessment provided us with information on high fall risk activities and with a method to differentiate fallers from non-fallers.

\section{Muscle strength assessment}

To determine whether fall prevalence relates to severity and pattern of muscle weakness, upper and lower limb muscle strength were evaluated by manual muscle testing according to the Medical Research Council (MRC) scale ${ }^{22}$ in eight frequent fallers, 10 infrequent fallers and 10 controls. Specifically, shoulder abduction and exorotation; elbow flexion and extension; wrist flexion and extension; hip ante- and retroflexion and $\mathrm{ab}$ and adduction; knee flexion and extension; and ankle plantar and dorsiflexion were tested.

\section{Data analysis}

The main outcome measures were self-reported frequency of falls, near falls and stumbles. Secondary outcome measures were self-reported circumstances and consequences of falls. The latter included injuries, fear of falling and reduction of activities due to fear of falling. Self-reported muscle weakness and the (in)ability to do certain functional tasks were compared with fall frequencies. Trunk sway angles in the roll (side-to-side) and pitch (for-aft) direction for the gait tasks and trunk angular velocities for the stance tasks were used as outcomes of the balance assessment. Differences between patients and controls were assessed using the unpaired Student $t$ test, ANOVA and Bonferroni, Mann-Whitney, $\chi^{2}$ and Kruskal-Wallis $\mathrm{H}$ in SPSS. Relative risk (RR) of falls was also calculated. Significance was set at $\mathrm{p}<0.05$.

\section{RESULTS}

\section{Prevalence of falls}

Falls occurred significantly more often in FSHD patients than in controls. In the retrospective questionnaire, $30 \%$ of the patients reported having at least one fall a month whereas none of the controls reported monthly falls (fig 1). Sixty-six per cent of the patients suffered at least monthly near falls compared with $6 \%$ of controls $(p<0.05)$. Stumbles occurred at least monthly in $57 \%$ of patients and in $9 \%$ of controls $(p<0.05)$.

During the 13 week prospective follow-up, 34 patients (47\%) reported 104 falls and four controls (8\%) reported 11 falls. The proportion of patients who reported at least one fall was much higher than that of controls (RR 5.8; 95\% confidence interval (CI) 2.2 to $15.3 ; \mathrm{p}<0.05)$. Also, a much higher proportion of patients $(26 \%)$ than controls (4\%) suffered multiple falls (RR $5.3 ; 95 \%$ CI 1.3 to $21.9 ; p<0.05)$. The RR of suffering at least one fall during follow-up when falls were reported in the questionnaire (at least monthly falls and/or monthly near falls) was 3.11 (95\% CI 1.48 to $6.54 ; \mathrm{p}<0.05$ ).

\section{Clinical relevance of falls}

Sixty-nine per cent of patients and $57 \%$ of controls reported sustaining one or more injuries from a fall at some point in time $(p=0.253)$ according to the retrospective questionnaire. Thirtyfour per cent of patients and $16 \%$ of controls reported severe injuries. Patients reported approximately 3.5 times more injuries due to falls than controls, while the proportion of patients (69\%) and controls (57\%) who had experienced an injurious fall hardly differed. This discrepancy can be explained by the number of multiple injuries in patients. Only $4.3 \%$ of controls suffered three or more injuries compared with $23 \%$ of
Figure 1 Retrospective falls in facioscapulohumeral disease (FSHD). Bar graphs depict the cumulative percentage of patients with FSHD and controls and their frequency of falls as reported in the questionnaire. Note that none of the controls reported a frequency greater than once a year. ${ }^{*} p<0.05$ FSHD versus controls.

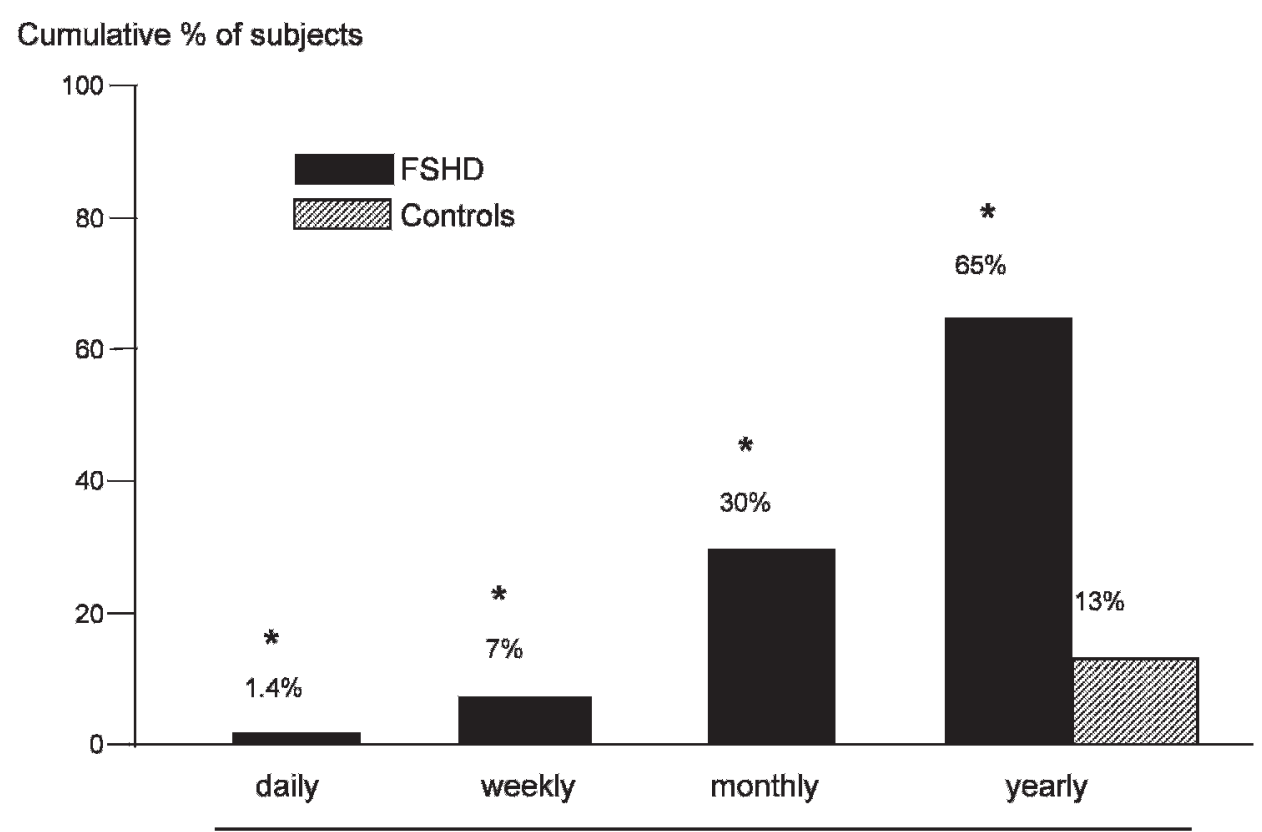

Frequency of falls 
Figure 2 Trunk sway during static and dynamic balance tasks. The upper three graphs $(A)$ represent sway angle traces in the roll (grey lines) and pitch (black lines) directions while climbing stairs for an individual frequent faller with facioscapulohumeral disease (FSHD), an infrequent FSHD faller and a healthy control subject. Bar graphs (B) represent the mean (SEM) of trunk sway angle for FSHD infrequent and frequent fallers and healthy controls when climbing stairs (B) and get up and go (C). For the stance tasks, standing on a firm surface with eyes closed (D) and standing on foam with eyes closed $(E)$, trunk angular velocity is given as a measure of balance. ${ }^{*} \mathrm{p}<0.05$, frequent fallers versus controls, $\$ \mathrm{p}<0.05$, frequent versus infrequent fallers. a. Individual traces climbing stairs

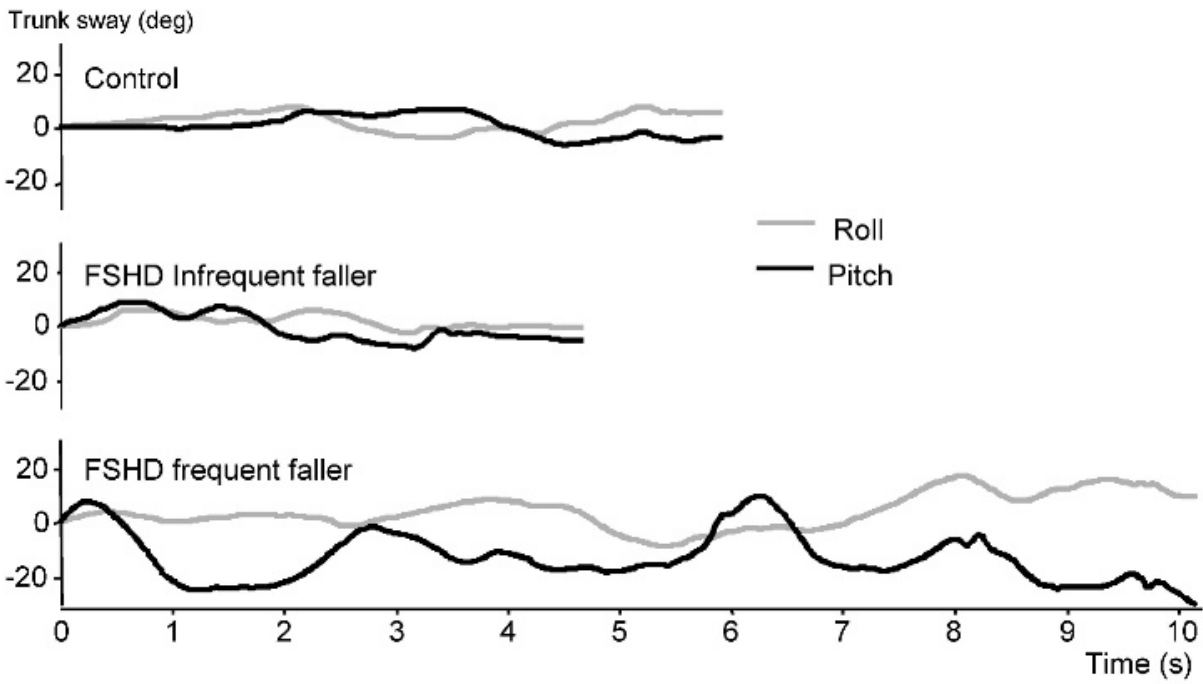

b. Climbing stairs

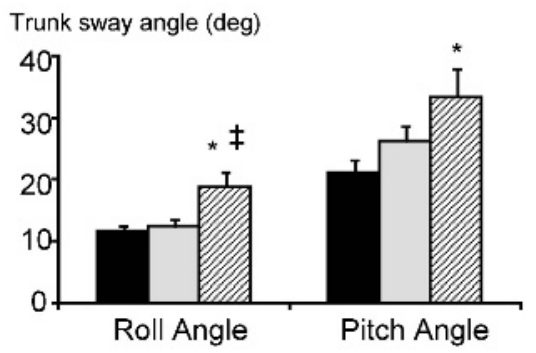

c. Get up and go

Trunk sway angle (deg)

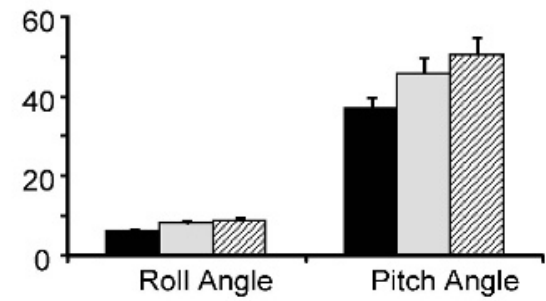

d. Standing on firm surface with eyes closed

e. Standing on foam with eyes closed Trunk angular velocity (deg/sec)
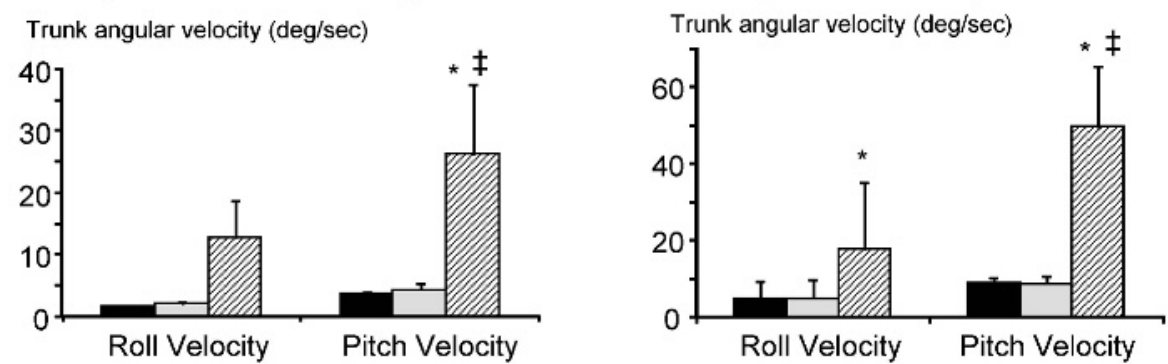

Control
FSHD infrequent fallers patients $(p<0.05)$. More than half of the patients $(51 \%)$ reported fear of falling compared with $7 \%$ of controls $(p<0.05)$. Due to their fear of falling, 35 patients $(49 \%)$ and two controls $(4 \%)$ reported reducing their participation in certain activities $(p<0.05)$. Most of the patients reported having stopped participating in sports and bicycling, and some reported having stopped walking, gardening, housework, shopping or leaving the house for pleasure activities. Control subjects only mentioned having stopped doing extreme sports, such as skiing.

During prospective follow-up, similar results were seen; $67 \%$ of the patients reported one or more injuries as a result of falling and six patients (18\%) reported multiple injurious falls. Controls all suffered at least one injurious fall and two reported multiple injurious falls. Major injuries that occurred among patients were fracture of the metacarpal bones, and three head skin wounds. One control suffered a rib contusion. All controls were able to get up by themselves after they had fallen whereas $24 \%$ of patients could not get up without help and $12 \%$ needed help occasionally. The results of one patient were missing.

\section{Fall circumstances}

The most common direction of falling could be specified by 39 patients in the questionnaire: $72 \%$ of these reported falling forwards. During follow-up, most patients (41\%) fell in a forward direction also. For controls, the direction of falls varied with each fall. In the questionnaire, most patients did not report a common time of day of falling (81\%). During follow-up, the time varied in $35 \%$ of patients: $26 \%$ reported falling in the afternoon, $21 \%$ in the morning and $9 \%$ in the evening. Only three controls specified a moment of falling, each at a different 

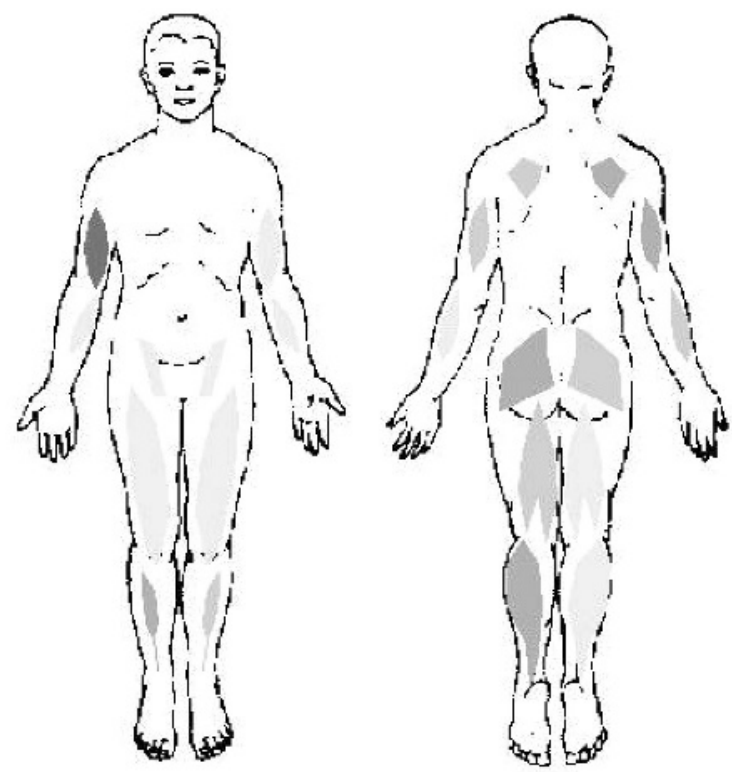

Right

Left Left

Right

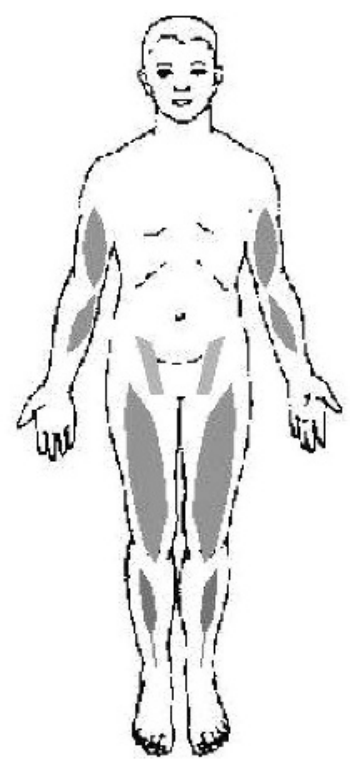

Right

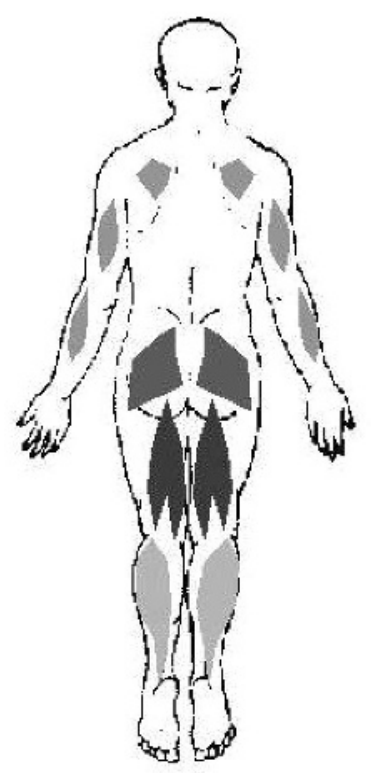

Right

Figure 3 Muscle weakness in infrequent and frequent fallers. The schematic figures depict the Medical Research Council (MRC) scores of several muscles (prime movers of the joint movement tested) for an individual infrequent and frequent faller with facioscapulohumeral disease (FSHD). ${ }^{23}$

time of day. In the questionnaire, 35 patients and 17 controls specified a location for their falls; $39 \%$ of these patients reported falling mostly at home while none of the controls did $(p<0.01)$. Also, during follow-up, the largest proportion of patients $(33 \%)$ always fell at home. During follow-up, most patients (41\%) suffered falls with an intrinsic cause. Controls always fell as a result of environmental hazards (six during skiing, three during ice skating, one on a slippery surface and one over the leg of a bed) compared with $32 \%$ of patients $(p<0.05)$.

\section{Pathophysiology}

\section{Characterising balance}

Two frequent fallers were unable to climb stairs and were omitted from evaluation. Figure $2 \mathrm{~A}$ shows individual trunk sway angle traces in the pitch and roll plane for a control subject, an infrequent FSHD faller and a frequent FSHD faller while climbing stairs. The frequent faller showed greater trunk

Table 2 Mean and median MRC scores for frequent and infrequent fallers

\begin{tabular}{llll}
\hline & $\begin{array}{l}\text { Controls } \\
(\mathbf{n}=10)\end{array}$ & $\begin{array}{l}\text { FSHD infrequent } \\
\text { fallers }(\mathbf{n}=10)\end{array}$ & $\begin{array}{l}\text { FSHD frequent } \\
\text { fallers }(\mathbf{n}=\mathbf{8})\end{array}$ \\
\hline MRC distal & & & \\
$\quad$ Mean (SEM) & $5.0(0.00)$ & $4.9(0.05)$ & $4.5(0.17)^{*}$ \\
$\quad$ Median & 5.0 & 5.0 & 4.7 \\
25th;75th percentiles & $5.0 ; 5.0$ & $4.8 ; 5.0$ & $4.2 ; 4.8$ \\
MRC proximal & & & \\
$\quad$ Mean (SEM) & $4,9(0.04)$ & $4,3(0.11) \dagger$ & $3.9(0.16)^{*}$ \\
$\quad$ Median & 5 & 4.3 & 3.9 \\
25th;75th percentiles & $4.8 ; 5.0$ & $4.1 ; 4.4$ & $3.7 ; 4.1$ \\
\hline
\end{tabular}

Mean (SEM) and median (25-75 percentiles) of the MRC scores for distal (ankle, wrist) and proximal (knee, hip, arm, shoulder) joint movements, to give an indication of the muscle weakness pattern of frequent and infrequent fallers.

${ }^{*} p<0.05$ compared with controls and infrequent fallers; $\uparrow p<0.05$ compared with controls.

FSHD, facioscapulohumeral disease; MRC, Medical Research Council. sway angle in the pitch and roll direction compared with the infrequent faller and the control subject. A significant difference in sway angle was found between the frequent fallers and controls in the pitch direction and in the roll direction between frequent fallers and the two other groups (fig 2B). There was no significant difference in task duration.

For get up and go, one frequent faller was unable to get up without help and was omitted from the analysis of this test. Trunk sway in the pitch and roll directions was lowest for the controls and highest for the frequent fallers (fig 2C). The differences in trunk sway between frequent fallers and controls were significant for roll velocity $(p<0.05)$ and pitch velocity $(p<0.05)$. No significant differences in task duration for this task were found or for the timed get up and go test.

Mean duration of standing on a firm support with eyes closed for frequent fallers was significantly shorter than for controls and infrequent fallers (14.5 s for frequent fallers vs $20 \mathrm{~s}$ for infrequent fallers and controls; $\mathrm{p}<0.05)$. The pitch sway velocity was significantly increased for frequent fallers compared with infrequent fallers and controls $(p<0.05)$ (fig 2D).

Significant differences in duration for standing on foam with eyes closed between frequent fallers and the two other groups (10.3 vs $20 \mathrm{~s} ; \mathrm{p}<0.01)$ were apparent. The sway angular velocities in controls and infrequent fallers were similar (fig 2E) but roll velocities were greater in frequent fallers compared with controls $(p<0.05)$, and pitch velocities were increased for frequent fallers compared with the two other groups $(p<0.05)$.

There were no significant differences between the three groups for the remaining tasks: standing on one leg, standing on foam with eyes open and tandem gait.

\section{Influence of muscle weakness on falls}

Figure 3 gives a schematic figure for the muscle weakness found in a frequent faller, who fell seven times during follow-up (fig 3A), and an infrequent faller, who did not fall during follow-up (fig 3B). 
Figure 4 Relative risks (RR) of falls, near falls and stumbles versus functional weakness. Plots represent RR and $95 \% \mathrm{Cl}$ for falls, near falls and stumbles for functional weakness and the various functional tasks reported in the questionnaire. Muscle weakness: "considerable" or "little to no" weakness, as reported in the questionnaire; Chair: ability to get up from a chair; Stairs: ability to walk stairs; Toes: ability to stand on the toes for $5 \mathrm{~s}$; Heels: ability to stand on heels for $5 \mathrm{~s}$. Each of these tasks was scored "with difficulty" or "with ease". Note the difference in scales for fall frequency versus near falls and stumble frequency. ${ }^{*} \mathrm{p}<0.05$.

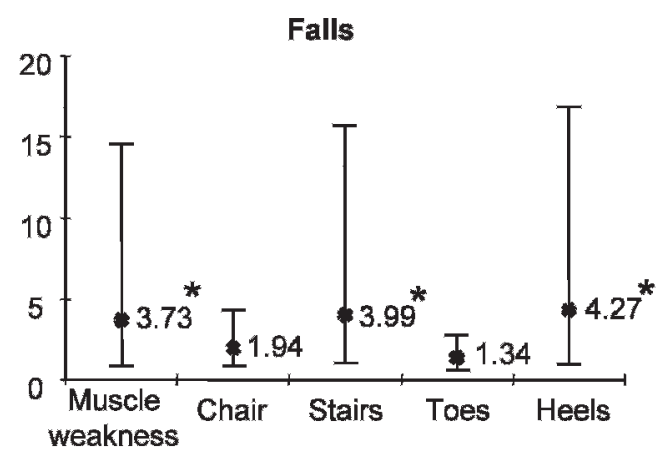

Near-falls

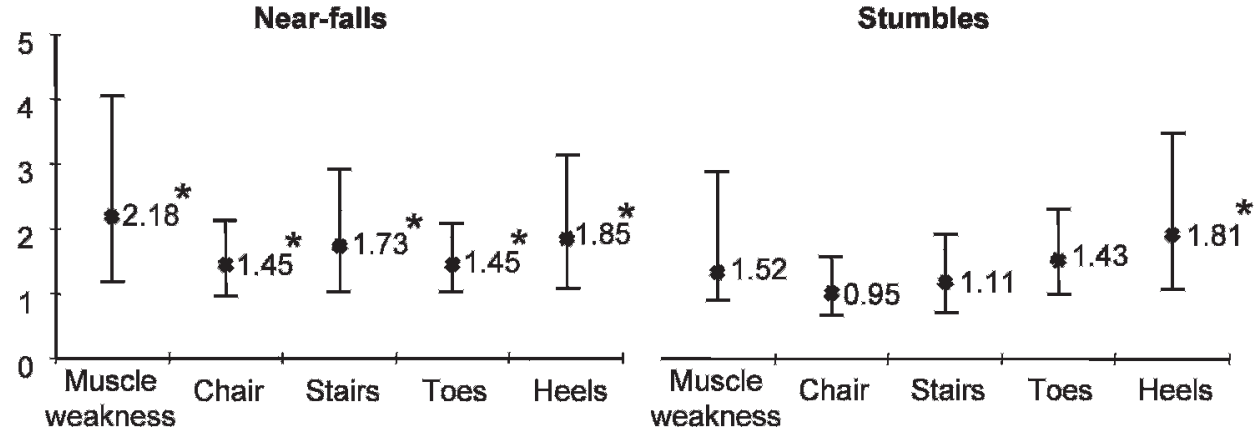

* RR 工 $95 \% \mathrm{Cl}$ interval $\perp$ 
Huntington's disease $e^{4}$ or the elderly $\left.{ }^{28}{ }^{29}\right)$. Patients and clinicians should be aware of this increased fall risk, and prevention strategies should focus on these specific activities. For example, handrails on stairs, or a stair elevator, might prevent falls. Moreover, balance measures might be a useful means of identifying FSHD patients at risk of falling. The lack of differences for the other tasks may be the result of greater variability in trunk sway; a consequence of increased difficulty of balance tasks within small groups. These tasks appeared unsuitable for distinguishing between FSHD fallers and infrequent fallers or controls in this study. But studies in a greater number of patients may reveal differences in more detail.

The influence of the pattern of muscle weakness (distal versus proximal) could not be differentiated in this patient population because many patients had both distal and proximal weakness. Moreover, we did not investigate the influence of asymmetry of muscle weakness on balance control. Studies in larger number of patients suffering from pure distal versus pure proximal weakness and with specific patterns of weakness (symmetric versus asymmetric) will increase knowledge on the influence of muscle weakness on the pathophysiology of falls.

It would be interesting to compare our findings with fall frequencies in other neuromuscular disease populations but this remains difficult because prospective epidemiological studies on falls in patients with neuromuscular disease are rare (reviewed by Horlings and colleagues ${ }^{11}$. Comparing rates of falls and the mechanisms leading to falls in different types of neuromuscular diseases may also shed light on the underlying pathophysiology, and further pinpoint the role of the peripheral nervous system in normal balance control.

\section{CONCLUSION}

In conclusion, falling is a common and clinically relevant problem among patients with FSHD. Falling is related to muscle weakness. Patients fall mainly due to intrinsic causes in a forward direction, and often fall at home. An increased fall risk can be expected during walking stairs, getting up from a chair and when the eyes are closed (or in the dark). Our findings highlight the clinical importance of falls in FSHD patients, the need for increased awareness of both the clinician and the patient in this matter, and the need for fall prevention strategies, for example to train patients in using different balance strategies.

Acknowledgements: We thank all patients and control subjects for participating in this study.

Funding: BRB was supported by NWO VIDI grant 016.076.352. JHJA was supported by the Swiss National Research Fund grant 32-117950/1.

\section{Competing interests: None.}

Ethics approval: The study was approved by the ethics committee of the Radboud University Nijmegen Medical Centre.

Provenance and peer review: Not commissioned; externally peer reviewed.

\section{REFERENCES}

1. Bloem BR, Grimbergen YA, Cramer $M$, et al. Prospective assessment of falls in Parkinson's disease. J Neurol 2001;248:950-8.

2. Pickering RM, Grimbergen $Y A$, Rigney $U$, et al. A meta-analysis of six prospective studies of falling in Parkinson's disease. Mov Disord 2007;15:1892-900.

3. Van de Warrenburg BP, Steijns JA, Munneke M, et al. Falls in degenerative cerebellar ataxias. Mov Disord 2005;20:497-500.

4. Grimbergen YAM, Knol MJ, Bloem BR, et al. Falls and gait disturbances in Huntington's disease. Mov Disord 2008;23:970-6.

5. Stolze $\mathbf{H}$, Klebe S, Zechlin C, et al. Falls in frequent neurological diseases-prevalence, risk factors and aetiology. J Neurol 2004;251:79-84.

6. Pieterse AJ, Luttikhold TB, de LK, et al. Falls in patients with neuromuscular disorders. J Neurol Sci 2006;251:87-90.

7. Friedman SM, Munoz B, West SK, et al. Falls and fear of falling: which comes first? A longitudinal prediction model suggests strategies for primary and secondary prevention. J Am Geriatr Soc 2002;50:1329-35.

8. Wiles CM, Busse ME, Sampson CM, et al. Falls and stumbles in myotonic dystrophy. J Neurol Neurosurg Psychiatry 2006;77:393-6.

9. Hopkinson ND, Hunt C, Powell RJ, et al. Inclusion body myositis: an underdiagnosed condition? Ann Rheum Dis 1993;52:147-51.

10. Stalenhoef $\mathbf{P}$, Crebolder HFJM, Knottnerus A, et al. Incidence, risk factors and consequences of falls among elderly subjects living in the community, a criteria-based analysis. J Clin Epidemiol 1997;7:328-34.

11. Horlings CGC, Van Engelen BGM, Allum JHJ, et al. A weak balance: the contribution of muscle weakness to postural instability and falls. Nature Clin Pract Neurol 2008;4:504-15

12. Moreland JD, Richardson JA, Goldsmith $\mathrm{CH}$, et al. Muscle weakness and falls in older adults: a systematic review and meta-analysis. J Am Geriatr Soc 2004:52:1121-9.

13. Lord SR, Allen GM, Williams $P$, et al. Risk of falling: predictors based on reduced strength in persons previously affected by polio. Arch Phys Med Rehabil 2002;83:757-63.

14. Padberg GWAM. Facioscapulohumeral disease. Thesis. Leiden: Leiden University, 1982.

15. Emery AE. Population frequencies of inherited neuromuscular diseases-a world survey. Neuromuscul Disord 1991;1:19-29.

16. Van der Maarel SM, Frants RR, Padberg GW. Facioscapulohumeral muscular dystrophy. Biochim Biophys Acta 2007;1772:186-94.

17. Pandya S, King WM, Tawil R. Facioscapulohumeral dystrophy. Phys Ther 2008;88:105-13.

18. Kalkman JS, Schillings ML, Zwarts MJ, et al. The development of a model of fatigue in neuromuscular disorders: a longitudinal study. J Psychosom Res 2007;62:571-9.

19. Van der Kooi EL. Facioscapulohumeral muscular dystrophy, interventions and intervention studies. Thesis. Nijmegen: Radboud University, 2006.

20. Ricci E, Galluzzi G, Deidda G, et al. Progress in the molecular diagnosis of facioscapulohumeral muscular dystrophy and correlation between the number of Kpnl repeats at the 4q35 locus and clinical phenotype. Ann Neurol 1999;45:751-7.

21. Allum JHJ, Carpenter MG. A speedy solution for balance and gait analysis: angular velocity measured at the centre of body mass. Curr Opin Neurol 2005;18:15-21.

22. MRC. Aids to the examination of the peripheral nervous system. London: Her Majesty's Stationary Office, 1976.

23. Pieterse AJ, Voermans NC, Tuininga $\mathrm{H}$, et al. Computer-aided visualization of muscle weakness distribution. J Neurol 2008;255:1670-8.

24. Bloem BR, Van Vugt JPP, Beckley DJ. Postural instability and falls in Parkinson's disease. Adv Neurol 2001;87:209-23.

25. Oude Nijhuis LB, Bloem BR, Carpenter MG, et al. Incorporating voluntary knee flexion into non-anticipatory balance corrections. J Neurophysiol 2007;98:3047-59.

26. Schillings ML, Kalkman JS, Janssen HMHA, et al. Experiences and physiological fatigue in neuromuscular disorders. Clin Neurophysiol 2007;118:292-300.

27. Nevitt MC, Cummings SR, Kidd S, et al. Risk factors for recurrent nonsyncopal falls. A prospective study. JAMA 1989;261:2663-8.

28. Buatois S, Gueguen R, Gauchard GC, et al. Posturography and risk of recurrent falls in healthy non-institutionalized persons aged over 65. Gerontology 2006;52:345-52

29. Melzer I, Benjuya N, Kaplanski J. Postural stability in the elderly: a comparison between fallers and non-fallers. Age Ageing 2004;33:602-7. 